\title{
Ergodic Type Bellman Equations of Risk-Sensitive Control
}

\author{
Kaise Hidehiro and Hideo Nagai
}

\section{Department of Mathematical Science, Graduate School of Engineering Science, Osaka University, Toyonaka, 560-8531, Japan}

\section{Abstract}

Ergodic type Bellman equations of risk-sensitive control in general cases are considered. We study the existence of solutions of the equations relating to the eigenvalue problem of Schödinger operators. Furthermore, by taking their singular limits, we obtain particular viscosity solutions of Hamilton-Jacobi-Isaacs equations of differential games which correspond to nolinear $H_{\infty}$-control problems.

\section{Introduction}

Let us consider a stochastic control problem with a risk-sensitive cost criterion on a finite time horizon

$$
I_{\epsilon}(T, x ; \theta, z)=\frac{\epsilon}{\theta} \log E_{x}\left[e^{\frac{\theta}{\epsilon} \int_{0}^{T} \Phi\left(X_{s}, z_{s}\right) d s}\right],
$$

where $X_{s}$ is a controlled process governed by the stochastic differential equation

$$
d X_{t}=\sqrt{\epsilon} \sigma\left(X_{t}\right) d B_{t}+\beta\left(X_{t}, z_{t}\right) d t, X_{0}=x,
$$

$z_{t}$ is a control process and $B_{t}$ is a standard $\mathcal{F}_{t}$ - Brownian motion process defined on a filtered probability space $\left(\Omega, \mathcal{F}, P ; \mathcal{F}_{t}\right)$. The constant $\theta \in R^{1} \backslash\{0\}$ is called risk-sensitive parameter. The meaning of the constant can be seen by looking at the asymptotic behaviour of the criterion as $\theta \rightarrow 0$ :

$$
\begin{aligned}
& I_{\epsilon}(T, x ; \theta, z) \\
& \quad \sim E\left[\int_{0}^{T} \Phi\left(X_{s}, z_{s}\right) d s\right] \\
& \quad+\frac{\theta}{\epsilon} \operatorname{Var}\left[\int_{0}^{T} \Phi\left(X_{s}, z_{s}\right) d s\right]+O\left(\theta^{2}\right)
\end{aligned}
$$

Namely, if $\theta>0$, then minimizing the criterion $I_{\epsilon}$ means to minimize the variance of the cost functional as well as its expected value. On the contrary, if $\theta<0$ it means to minimize the expected value but maximize its variance. In that sense we call the case where $\theta>0$ $(\theta<0$ respectively) risk averse (resp. risk seeking). We define the value function of the problem as

$$
w_{\epsilon}(t, x)=\inf _{z .} I_{\epsilon}(T-t, x ; \theta, z),
$$

where $z_{t}$ ranges over the set of all $\mathcal{F}_{t}$ - progressively measurable processes taking its value on a Borel subset $Z$ of $R^{N_{1}}$. The value function $w_{\epsilon}(t, x)$ is seen to formally satisfy the Bellman equation

$$
\begin{cases}\frac{\partial w_{\epsilon}}{\partial t}+\frac{\epsilon}{2} a^{i j} D_{i j} w_{\epsilon}+Q_{0}\left(x, \nabla w_{\epsilon}\right) & =0 \\ w_{\epsilon}(T, x) & =0\end{cases}
$$

where

(1.4) $Q_{0}(x, p)=\frac{\theta}{2} a^{i j} p_{i} p_{j}+\inf _{z \in Z}\left\{\beta^{i}(x, z) p_{i}+\Phi(x, z)\right\}$.

Here we utilized summation convention. We note that cost function $\Phi(x, z)$ may be assumed to be nonnegative and go to $\infty$ as $|x|$ or $|z|$ tends to $\infty$ when we consider generalizing LEQG (Linear Exponential Quadratic Gaussian) case, namely the case where $\beta(x, z)=A x+B z, \sigma(x)=\sigma, \Phi(x, z)=$ $(1 / 2) x^{*} R x+(1 / 2) z^{*} S z$. Therefore, if $\theta>0$, it may occur that the criterion $I_{\epsilon}(T, x ; \theta, z)$ diverges for any control $z$. In such a case we say the problem breaks down. It might be very important to know the conditions under which no breakdown occurs and they have been studied in [8],[22]. Existence of solutions of Bellman equation of parabolic type (1.3) has been also proven under the conditions there.

Now, under the conditions in which no breakdown occurs for each $T>0$, we can set up the problems on infinite time horizon and in the present paper we shall study Bellman equations of ergodic type in relation to the risk-sensitive control problems on infinite time horizon and consider their singular limits as an extension of [19]. As was done in [19] we shall first discuss existence and uniqueness of the solution of the equation

$$
\chi_{\epsilon}=\frac{\epsilon}{2} a^{i j} D_{i j} v_{\epsilon}+Q_{0}\left(x, \nabla v_{\epsilon}\right)
$$

and then study its singular limit as $\epsilon \rightarrow 0$. Note that in such a equation as (1.5) we consider the the pair of a function $v_{\epsilon}$ and a constant $\chi_{\epsilon}$ a solution. (1.5) is thought to be the limit equation of (1.3) as $T \rightarrow \infty$. More precisely, under suitable conditions, the solution $v_{\epsilon}$ (respectively $\chi_{\epsilon}$ ) of (1.5) can be obtained as the limit of $w_{\epsilon}(0, x)-w_{\epsilon}\left(0, x_{0}\right)$ (respectively 
$\left.\left(\partial w_{\epsilon} / \partial t\right)(0, x)\right)$ as the time horizon $T \rightarrow \infty$, where $x_{0}$ is a fixed particular point (cf.[22] ). From a probabilistic view point, $\chi_{\epsilon}$ is considered to be the constant characterizing the minimum value of the averaging cost criterion of the risk-sensitive control problem on infinite time horizon:

$$
\zeta_{\epsilon}=\liminf _{T \rightarrow \infty} \frac{1}{T} \frac{\epsilon}{\theta} \log E\left[e^{\frac{\theta}{\epsilon} \int_{0}^{T} \Phi\left(X_{s}, z_{s}\right) d s}\right],
$$

where $X_{s}$ is a controlled stochastic process governed by the above stochastic differential equation (1.2) and it is regarded as the following Bellman-Isaacs equation

$$
\begin{aligned}
\chi_{\epsilon}= & \frac{\epsilon}{2} a^{i j} D_{i j} v_{\epsilon}+\inf _{z}\left\{\beta^{i}(x, z) D_{i} v_{\epsilon}+\Phi(x, z)\right\} \\
& +\sup _{y \in R^{N}}\left\{y^{i} D_{i} v_{\epsilon}-\frac{1}{2 \theta} a_{i j}^{-1} y^{i} y^{j}\right\} .
\end{aligned}
$$

It is important to change to such a form from the viewpoint considering the relationships between risk sensitive control and $H_{\circ \circ}$ control. In fact, when letting $\epsilon$ tend to 0 , we have the limit equation under suitable conditions

$$
\begin{aligned}
\chi_{0}= & \inf _{z}\left\{\beta(x, z)^{i} D_{i} v+\Phi(x, z)\right\} \\
& +\sup _{y \in R^{N}}\left\{y^{i} D_{i} v-\frac{1}{2 \theta} a_{i j}^{-1} y^{i} y^{j}\right\}
\end{aligned}
$$

and the pair of the constant $\chi_{0}$ and the function $v$ is considered to be a solution of the equation and to be connected to a differential game relevant to nonlinear $H_{\infty}$ control. Such relationship has been first noticed by P. Whittle ([26],[27] cf. also [24]) by heuristic arguments and since then lots of work have been done on the matter to justify his arguments. First success has been done by James [15] and Fleming-McEneaney [11] independently in parabolic case (namely finite time horizon case) with at most linear growth $\Phi$, where LEQG case is not included, though. The considerably general case including LEQG has been studied in [7], which is largely extended in [5] later. The problems of infinite time horizon have been studied by [12],[21] and [13],[22]. Our results [18],[19] and the present paper are extension of their works from analytical view points. In particular, they are based on the relationships between the ergodic type Bellman equations and the eigenvalue problems of Schroödinger operators, which have been noticed in [6] and [13]. There are also several attempts to treat partially observable case (cf. [3], [17] etc.). On the other hand, irrelevant to small noise limit, risk sensitive control problems have been studied so far from long time ago (cf. [9], [14], [25],[10], etc.). Among those we note the recent work of Bielecki-Pliska [10] related to their application to the problems on mathematical finance, where they actually applied the results of [22] to optimal portfolio problems. It may give rise to new development of the study of risk sensitive control.

As noted above, to study equation (1.5), we need assumptions ensuring no breakdown. So, to specify such situation, in considering the case where

$$
\beta^{i}(x, z)=-a^{i j}(x) D_{j} U(x)+\frac{\epsilon}{2} D_{j} a^{i j}(x)+c^{i}(x, z),
$$

and

$$
\Phi(x, z)=V(x)+\phi(x, z),
$$

we shall make main assumptions relating to growth orders of the functions $U$ and $V$, and the size of the risk-sensitive parameter $\theta$.

We actually consider the following more general equation

$$
\begin{aligned}
\chi_{\epsilon}= & \frac{\epsilon}{2} D_{i}\left(a^{i j} D_{j} v\right) \\
& -(\nabla U)^{*} a \nabla v+Q(x, \nabla v)+V,
\end{aligned}
$$

under the main assumptions that:

there exists a constant $\kappa>0$ and a locally bounded function $K(x)$ such that

$$
\begin{aligned}
\tilde{V}_{\epsilon} \equiv & \frac{1}{2}(\nabla U)^{*} a \nabla U \\
& -\kappa(V+K)-\frac{\epsilon}{2} D_{i}\left(a^{i j} D_{j} U\right) \rightarrow \infty
\end{aligned}
$$

as $|x| \rightarrow \infty, \epsilon \leq 1$, and that there exists a constant $\kappa_{0}>0$ and a locally bounded function $K_{0}(x)$ such that $\forall x, p \in R^{N}$

$$
\frac{\kappa_{0}}{2} p^{*} a p-K_{0}(x) \leq Q(x, p) \leq \frac{\kappa}{2} p^{*} a p+K(x) .
$$

After getting a solution of equation (1.8) we deduce its limit equation as $\epsilon \rightarrow 0$ :

$$
-(\nabla U)^{*} a \nabla v-Q(x, \nabla v)-V-\chi_{0}=0
$$

by using viscosity methods.

In equations such as (1.8) quadratic growth nonlinear term $Q(x, p)$ with respect to $p$ plays a crucial role. Though the case where $Q(x, p)$ has negative quadratic growth in $p$, ergodic type Bellman equations have been dealt with in considerably general settings by Bensoussan-Frehse [4], if $\theta$ is large enough in a specified case where $Q(x, p)$ is defined by the formula (1.4), then $Q(x, p)$ may have positive quadratic growth with respect to $p$ as we shall see below in a typical case.

\section{Example 1}

Assume $Z=R^{N_{1}}, c(x, z)=b(x)+C(x) z$ and $\phi(x, z)=(1 / 2) z^{*} S(x) z$, where $C(x)$ is $N \times N_{1}$-matrix and $S(x)$ is $N \times N$-symmetric, positive-definite matrix. Then we can calculate the infimum of $Q(x, p)$ as follows.

$$
Q(x, p)=b(x)^{*} p+\frac{1}{2} p^{*}\left(\theta a(x)-C(x) S(x)^{-1} C(x)^{*}\right) p .
$$

And we see that the sign of the quadratic form $Q(x, p)$ depends on the size of $\theta$.

On the other hand, we have studied in a particular case the equations admitting large $\theta$ in the previous 
work [18] and extended it to more genaral case in [19]. In the present paper we remark that by using the same methods as [19] we can slightly generalize the results obtained there as above. The reason why we discuss the case of large $\theta$ lies in considering relationships between risk-sensitive control and nonlinear $H_{\infty}$-control as noted above. In LEQG case, the constant $1 / 2 \theta$ corresponds to disturbance attenuation level in $\mathrm{H}_{\infty^{-}}$ control theory and the possible lower bound might be one of main concern in the theory.

Finally we illustrate various examples.

Example 2 In (1.3) we assume that $a^{i j}(x)$ is constant matrix and $\beta(x, z)=A x+B z, \Phi(x, z)=\frac{1}{2} x^{*} R x+$ $\frac{1}{2} z^{*} S z, Z=R^{N_{1}}$, where $A, B, R, S$ are constant matrices such that $R$ is nonnegative definite and $S$ positive definite symmetric. Then the solution of (1.3) has the following explicit form:

$$
w_{\epsilon}(t, x)=\frac{1}{2} x^{*} P(t) x+\frac{1}{2} G_{\epsilon}(t),
$$

where $P(t)$ is a solution of matrix Riccati differential equation:

$$
\begin{aligned}
& \dot{P}(t)+P(t) A+A^{*} P(t) \\
& \quad-P(t)\left(B S^{-1} B^{*}-\theta a\right) P(t)+R=0, \\
& P(T)=0
\end{aligned}
$$

and $G_{\epsilon}(t)$ the one of differential equation:

$$
\dot{G}_{\epsilon}(t)+\frac{\epsilon}{2} \operatorname{tr}(P(t) a)=0, \quad G_{\epsilon}(T)=0 .
$$

It is known that under suitable conditions, as $T \rightarrow \infty$, $P(0)=P(0 ; T)$ converges to a nonnegative definite symmetric matrix $P$ which is a solution of algebraic Riccati equation:

$$
P A+A^{*} P-P\left(B S^{-1} B^{*}-\theta a\right) P+R=0
$$

and therefore $\dot{G}_{\epsilon}$ to $-\frac{\epsilon}{2} \operatorname{tr} P a$. Thus we obtain the solution $\left(v_{\epsilon}, \chi_{\epsilon}\right)=\left(\frac{1}{2} x^{*} P x, \frac{\epsilon}{2} \operatorname{tr} P a\right)$ of ergodic type Bellman equation (1.5). In this case (1.7) is nothing but

$$
x^{*}\left[P A+A^{*} P-P\left(B S^{-1} B^{*}-\theta a\right) P+R\right] x=0 .
$$

Example 3 In (1.2) and (1.3) we consider the case where $\sigma \equiv I, \beta(x, z)=z$ and $\Phi(x, z)=V(x)+\frac{1}{2}|z|^{2}$, $Z=R^{N}$. We assume that $V(x) \geq 0$ and $V(x) \rightarrow$ $\infty$, as $|x| \rightarrow \infty$. In this case ergodic type equation (1.5) turns out to be

$$
\lambda_{\epsilon}=\frac{\epsilon}{2} \Delta v_{\epsilon}-\frac{1-\theta}{2}\left|\nabla v_{\epsilon}\right|^{2}+V(x)
$$

where $\lambda_{\epsilon}$ is the principal eigen value of the Schrödinger operator $\frac{\epsilon}{2} \Delta-\frac{1}{\epsilon} V$ on $L^{2}\left(R^{N}\right)$. To study the ergodic type equation we take up discounted type equation

$$
\alpha v_{\alpha, \epsilon}=\frac{\epsilon}{2} \Delta v_{\alpha, \epsilon}-\frac{1-\theta}{2}\left|\nabla v_{\alpha, \epsilon}\right|^{2}+V(x)
$$

and as a limit of this equation as $\alpha \rightarrow 0$ we shall obtain ergodic type eqution (cf.[6]). Our approch to Theorem 3.1 is based on this example. To study the limit as $\epsilon \rightarrow 0$ is related to semiclassical analysis in Mathematical physics (cf.[13]).

Example 4(Bielecki-Pliska) According to BieleckiPliska we shall consider a factor model appearing mathematical finance. Prices of $m$ securities are defined by the stochastic differential equation:

$$
\begin{aligned}
& d S^{i}(t)=S^{i}(t)\left(a+A X_{t}\right)^{i} d t+\sum_{k=1}^{n+m} \sigma_{k}^{i} d W_{t}^{k}, \\
& S^{i}(0)=s^{i}, \quad i=1, \ldots, m
\end{aligned}
$$

and factor process by

$$
d X_{t}=\left(b+B X_{t}\right) d t+\Lambda d W_{t}, \quad X(0)=x \in R^{n},
$$

The value process $V_{t}=V_{t}(h)$ when an investor chooses the portofolio $h=\left(h^{i}(t)\right)_{i=1}^{m}$ among the securities is defined by

$$
\begin{aligned}
\frac{d V_{t}}{V_{t}} & =\sum_{i=1}^{m} h^{i}(t) \frac{d S^{i}(t)}{S^{i}(t)} \\
& =\sum_{i=1}^{m} h^{i}(t)\left\{\left(a+A X_{t}\right)^{i} d t+\sum_{k=1}^{m+n} \sigma_{k}^{i} d W_{t}^{k}\right\} \\
V_{0} & =v
\end{aligned}
$$

Their problem is to maximize the risk-sensitized expected growth rate of the value:

$$
\chi=\sup _{h .} \liminf _{T \rightarrow \infty}-\frac{2}{\theta} \frac{1}{T} \log E\left[e^{(-\theta / 2) \log V_{T}(h)}\right]
$$

The constant $\chi$ is characterized by ergodic type Bellman equation :

$$
\begin{aligned}
& \chi=\frac{1}{2}\left(\Lambda \Lambda^{*}\right)^{i j} D_{i j} v+(b+B x)^{i} D_{i} v \\
& -\frac{\theta}{4}\left(\Lambda \Lambda^{*}\right)^{i j} D_{i} v D_{j} v K_{\theta}(x)
\end{aligned}
$$

where $\left.K_{\theta}=\inf _{h} \frac{1}{2}(\theta / 2)+1\right) h^{*} \Sigma \Sigma^{*} h-h^{*}(a+A x)$. Moreover optimal portforio is defined by the solution of the ergodic type equation.

\section{Discounted type equation}

In the present section we shall consider existence of the nonnegative solution of the following Bellman equation of discounted type on the whole Euclidean space $R^{N}$ arising from risk-sensitive control :

$$
\begin{aligned}
& -\frac{\epsilon}{2} D_{i}\left(a^{i j} D_{j} u_{\alpha}\right)+(\nabla U)^{*} a \nabla u_{\alpha} \\
& \quad-Q\left(x, \nabla u_{\alpha}\right)-V+\alpha u_{\alpha}=0, \alpha>0 .
\end{aligned}
$$

$Z$ is a control region, a Borel subset of $R^{N_{1}}$, and $\theta \in$ $R \backslash\{0\}$ is a risk-sensitive parameter. We are interested in the size of $\theta$ ensuring existence of the solution of (2.1). We make the assumptions as follows. 
(A 1)

$$
U, V \geq 0
$$

(A 2) there exists a constant $\kappa>0$ and a locally bounded function $K(x) \geq 0$ such that

$$
\begin{aligned}
\tilde{V}_{\epsilon} \equiv & \frac{1}{2}(\nabla U)^{*} a \nabla U-\kappa(V+K) \\
& -\frac{\epsilon}{2} D_{i}\left(a^{i j} D_{j} U\right) \rightarrow \infty
\end{aligned}
$$

as $|x| \rightarrow \infty, \epsilon \leq 1$,

(A 3) there exists a constant $\kappa_{0}>0$ and locally bounded function $K_{0}(x)$ such that

$$
\frac{\kappa_{0}}{2} p^{*} a p-K_{0}(x) \leq Q(x, p) \leq \frac{\kappa}{2} p^{*} a p+K(x),
$$

$\forall x, p \in R^{N}$, where $\kappa$ is the same constant as (A 2),

(A 4) for $0<\nu_{1}<\nu_{2}, a(x)$ satisfies

$$
\nu_{1}|p|^{2} \leq p^{*} a p \leq \nu_{2}|p|^{2}, \forall x, p \in R^{N},
$$

(A 5)

$$
\begin{aligned}
& \left|Q_{p}(x, p)\right| \leq K_{1}(x)+K_{2}(x)|p| \\
& \left|Q_{x}(x, p)\right| \leq \hat{K}_{1}(x)+\hat{K}_{2}(x)|p|^{2}, \forall x, p \in R^{N}
\end{aligned}
$$

where, $K_{1}, K_{2}, \hat{K}_{1}$ and $\hat{K}_{2}$ are locally bounded functions on $R^{N}$,

(A 6) $a, U, V$ and $Q(x, p)$ are smooth functions,

(A 7 ) there exists a constant $M$ such that

$$
U(x) \leq M \tilde{V}_{\epsilon}(x),|x| \gg 1,
$$

(A 8)

$$
e^{-U} \in L^{2}\left(R^{N}\right),|\nabla U|^{2} e^{-2 U} \in L^{\infty}\left(R^{N}\right),
$$

(A 9)

$$
e^{-\delta \sqrt{\bar{V}}_{\epsilon}} \in L^{2}\left(R^{N}\right), \delta>0
$$

where $\bar{V}_{\epsilon}=\tilde{V}_{\epsilon}-\min \tilde{V}_{\epsilon}$.

Under the above assumptions we first construct a super-solution $\gamma_{\alpha}$ of the equation (2.1) and for that we consider the following equation.

$$
\begin{aligned}
& -\frac{\epsilon}{2} D_{i}\left(a^{i j} D_{j} \gamma_{\alpha}\right)+(\nabla U)^{*} a \nabla \gamma_{\alpha} \\
& -\frac{\kappa}{2}\left(\nabla \gamma_{\alpha}\right) a \nabla \gamma_{\alpha}-(V+K)+\alpha \gamma_{\alpha}=0 .
\end{aligned}
$$

If (2.2) has a solution, it turns out to be a supersolution of (2.1) because of assumption (A3). To study equation (2.2), we take a transformation

$$
\eta_{\alpha}=e^{(\kappa / \epsilon) \gamma_{\alpha}-(1 / \epsilon) U}
$$

and obtain the following equation

$$
-\frac{\epsilon}{2} D_{i}\left(a^{i j} D_{j} \eta_{\alpha}\right)+\frac{1}{\epsilon} \tilde{V}_{\alpha} \eta_{\alpha}=-\alpha \eta_{\alpha} \log \eta_{\alpha}
$$

where $\tilde{V}_{\alpha}=\tilde{V}_{\epsilon}+\alpha U$. As for (2.3) we can see in the same way as [19] existence of the unique solution in a suitable Sobolev space, from which we obtain the sulution $\gamma_{\alpha}$ of (2.2) by setting

$$
\gamma_{\alpha}=\frac{\epsilon}{\kappa} \log \eta_{\alpha}+\frac{1}{\kappa} U
$$

Furhtermore let us set for a constant $\lambda>0$,

$$
\zeta=e^{-\lambda / \epsilon-U / \epsilon}
$$

Then $\zeta$ satisfies

$$
\begin{aligned}
-\frac{\epsilon}{2} D_{i}\left(a^{i j} D_{j} \zeta\right)+\frac{1}{\epsilon} \tilde{V}_{\alpha} \zeta= & -\frac{\kappa}{\epsilon}(V+K) e^{-\lambda / \epsilon-U / \epsilon} \\
& +\frac{\alpha U}{\epsilon} e^{-\lambda / \epsilon-U / \epsilon} .
\end{aligned}
$$

Since

$$
-\alpha \zeta \log \zeta=(\alpha \lambda / \epsilon) e^{-\lambda / \epsilon-U / \epsilon}+(\alpha U / \epsilon) e^{-\lambda / \epsilon-U / \epsilon}
$$

and $V$ is nonnegative we have

$$
-\frac{\epsilon}{2} D_{i}\left(a^{i j} D_{j} \zeta\right)+\frac{1}{\epsilon} \tilde{V}_{\alpha} \zeta \leq-\alpha \zeta \log \zeta
$$

and so $\zeta$ turns out to be a sub-solution of (2.3). Thus we can see in the same way as [19] that $\zeta \leq \eta_{\alpha} \equiv$ $e^{\frac{\kappa}{\epsilon} \gamma_{\alpha}-\frac{U}{\epsilon}}$, which implies that $-\lambda \leq \kappa \gamma_{\alpha}$ for each $\lambda>0$. Hence we see that $\gamma_{\alpha} \geq 0$. So, we obtain the following lemma.

Lemama 2.1 Under assumptions (A1), (A2),(A4), (A6), (A8) and (A9) the solution $\gamma_{\alpha}$ of (2.2) obtained by formula (2.4) satisfies $\gamma_{\alpha} \geq 0$.

We next consider the following Dirichlet problem

$$
\begin{gathered}
-\frac{\epsilon}{2} D_{i}\left(a^{i j} D_{j} u\right)+(\nabla U)^{*} a \nabla u \\
-Q(x, \nabla u)-V+\alpha u=0 \text { in } B_{R}(0) \\
u=\gamma_{\alpha} \text { on } \partial B_{R}(0)
\end{gathered}
$$

in the ball $B_{R}(0)$ with the radius $R$ centered at 0 . Owing to Theorem 8.3, Chapter 4 in [20], we see that Dirichlet problem (2.6) and (2.7) has a unique solution $u=u_{\alpha, R} \in C^{2, \gamma}\left(\bar{B}_{R}\right)$. We furthermore have the following estimates.

Lemma 2.2 Under assumptions (A1) - (A4), (A6), (A8) and (A9) we have the estimates for the solution $u_{\alpha, R}$ of $(2.8)$

$$
0 \leq u_{\alpha, R} \leq \gamma_{\alpha} \text { in } \bar{B}_{R}(0) .
$$


Now as the limit of the solution $u_{\alpha, R}$ as $R \rightarrow \infty$ we obtain the solution $u_{\alpha}$ of Bellman equation of discounted type (2.1), after getting gradient estimates of $u_{\alpha, R}$ by using Bernstein's methods.

Theorem 2.1 Under assumptions (A1) - (A9) there exists a solution $u_{\alpha} \in C^{2}\left(R^{N}\right)$ of the equation (2.1) such that

$$
0 \leq u_{\alpha} \leq \gamma_{\alpha}
$$

Moreover the solution satisfies for each $r>0$

$$
\left\|\alpha u_{\alpha}\right\|_{L^{\infty}\left(B_{r}\right)} \leq K_{r}
$$

and

$$
\left\|\nabla u_{\alpha}\right\|_{L^{\infty}\left(B_{r}\right)} \leq K_{r}
$$

where $K_{r}$ is a constant independent of $\alpha$.

\section{$3 \quad$ Ergodic type equation}

Now we shall deduce the Bellman equation of ergodic type :

$$
\begin{gathered}
-\frac{\epsilon}{2} D_{i}\left(a^{i j} D_{j} v_{\epsilon}\right)+(\nabla U)^{*} a \nabla v_{\epsilon} \\
-Q\left(x, \nabla v_{\epsilon}\right)-V+\chi_{\epsilon}=0,
\end{gathered}
$$

from discounted type equation (2.1). We actually have the following theorem.

Theorem 3.1 Let us set $v_{\alpha}(x)=u_{\alpha}(x)-u_{\alpha}\left(x_{0}\right)$ for an arbitrary fixed point $x_{0}$. Then, under assumptions (A1) - (A9) $v_{\alpha}$ converges to a $C^{2}$-function $v_{\epsilon}$ strongly in $H_{\text {loc }}^{1}\left(R^{N}\right)$ and also uniformly on each compact set as $\alpha \rightarrow 0$, and $\alpha u_{\alpha}$ to a constant $\chi_{\epsilon}$ uniformly on each compact set, by taking a subsequence if necessary. Moreover, the pair $\left(v_{\epsilon}, \chi_{\epsilon}\right)$ satisfies Bellman equation of ergodic type (3.1) and $-(\kappa / \epsilon) v_{\epsilon}+(1 / \epsilon) U$ is bounded from below.

Now let us consider uniqueness of the solution of (3.1) Assume that $Q(x, p)=b(x)^{*} p+(1 / 2) p^{*} A(x) p$, where $A(x)=\left(A^{i j}(x)\right)$ is a symmetric matrix satisfying $\left(\kappa_{0}-\delta_{0}\right) a(x) \leq A(x) \leq(\kappa-\delta) a(x)$ for some $\delta_{0}, \delta>0$. Note that in the present case assumptions (A 2) and (A 3) is to be set as $K(x)=$ $\delta^{-1} b^{*}(x) a^{-1} b(x)$ and $K_{0}(x)=\delta_{0}^{-1} b^{*}(x) a^{-1}(x) b(x)$.

Theorem 3.2 Assume the assumptions of Theorem 3.1 in the above mentioned settings. If $\left(v_{\epsilon, i}, \chi_{\epsilon, i}\right)$, $i=1,2$ are solutions such that $-(\kappa / \epsilon) v_{\epsilon, i}+(1 / \epsilon) U$ is bounded below for each $i$, then $\chi_{\epsilon, 1}=\chi_{\epsilon, 2}$ and $v_{\epsilon, 1}(x)=v_{\epsilon, 2}(x)+c$ for some constant $c$.

\section{Singular limits}

Now we shall consider asymptotic behavior of the solutions of equation (3.1) as $\epsilon \rightarrow 0$.

Theorem 4.1 Let $\left(v_{\epsilon}, \chi_{\epsilon}\right)$ be a solution of (3.1) obtained in Theorem 3.1. Then, $v_{\epsilon}$ converges to a function $v$ uniformly on each compact set and $\chi_{\epsilon}$ to a constant $\chi_{0}$, by taking a subsequence if necessary. Moreover, $v$ is a viscosity solution of

$$
(\nabla U)^{*} a \nabla v-Q(x, \nabla v)-V+\chi_{0}=0
$$

and $\chi_{0}$ satisfies estimate

$$
0 \leq \chi_{0} \leq-\frac{1}{\kappa} \min \left(\frac{1}{2}(\nabla U)^{*} a \nabla U-\kappa(V+K)\right) \text {. }
$$

As for uniqueness of the solution of (4.1), we have the following.

Theorem 4.2 Let $Q(x, p)$ be the one of Theorem 3.2 and $\hat{V}(x)=(1 / 2)(\nabla U)^{*} a \nabla U-\kappa\left(V+\delta^{-1} b^{*} a^{-1} b\right)$ and we assume that there exists $x_{0} \in R^{N}$ such that $\hat{V}(x)>$ $\hat{V}\left(x_{0}\right)=0, \forall x \neq x_{0}$ and $\hat{V}(x) \rightarrow \infty$. Then, the viscosity solution $v$ of $(4.1)$ such that $\hat{v} \equiv-v+(1 / \kappa) U$ is bounded from below and $\hat{v}\left(x_{0}\right)=0$ is unique.

From the uniqueness result for (4.1), we obtain the following corollary in the same way as the proof of Corollary 4.1 of [13]. Note that we have $\chi_{0}=0$ because of (4.2) and the assumption of Theorem 4.2.

Corollary 4.1 Besides assumptions (A1) - (A9) we assume the assumptions of Theorem 4.2. Let $\left(v_{\epsilon}, \chi_{\epsilon}\right)$ be a solution of (3.1) obtained of Theorem (3.1) which is normalized as $v_{\epsilon}\left(x_{0}\right)=0$. Then, $v_{\epsilon}$ converges to the unique viscosity solution $v$ of (4.1) with $Q(x, p)=$ $b(x)^{*} p+(1 / 2) p^{*} A(x) p$ uniformly on each compact set and $\chi_{\epsilon}$ to 0 .

\section{Conclusion}

By treating general ergodic type Bellman eqautions of risk-sensitive control with large risk-sensitive parameters and their singular limits the relationships between nonlinear $H_{\infty}$ control and risk-sensitive control have been extensively generalized.

\section{References}

[1] T. Basar and P. Bernhard, " $H^{\infty}$ - Optimal Control and Related Minimax Design Problems", Birkhauser, Boston, (1991)

[2] A. Bensoussan and R.J. Elliott, "A finite dimensional risk sensitive control problem", SIAM J. Control and Optim. vol.33,1834/1846 (1995), 
[3] A. Bensoussan and R.J. Elliott, "General FiniteDimensional Risk-Sensitive Problems and Small Noise Limits", IEEE Trans. Aut. Control, vol.41,210/215 (1996)

[4] A. Bensoussan and J. Frehse, On Bellman equations of ergodic control in $R^{N}$, J. Reine Angew. Math. 429,125/160 (1992) .

[5] A. Bensoussan, J. Frehse and H. Nagai, Some results on risk-sensitive control with full observation, Applied Mathematics and Optimization, Vol. 37,1/41 (1998).

[6] A. Bensoussan and H. Nagai, An ergodic control problem arising from the principal eigenfunction of an elliptic operator, Math. Soc. Japan, Vol. 43, No. 1,49-65 (1991)

[7] A. Bensoussan and H. Nagai, "Min-max characterization of a smaal noise limit on risk sensitive control", SIAM J. Control and Optim., vol. 35,1093-1115 (1997)

[8] A. Bensoussan and H. Nagai, Conditions for no breakdown and Bellman equations of risksensitive control, to appear in Applied Mathematics and Optimization.

[9] A. Bensoussan and J.H. Van Schuppen, "Optimal control of partially observable stochastic systems with an exponential-of integral performance index", SIAM J. Cont. Optim., vol. 23, 599/613 (1985)

[10] T.R. Bielecli and S.R. Pliska, "Risk-Sensitive Dynamic Asset Management", Appl. Math. Optim. vol. $39,337 / 360(1999)$

[11] W.H. Fleming and W.M. McEneaney, "Risk sensitive optimal control and differential games", Lecture Note in Control and Inform. Sci., vol. $184,185 / 197$ (1992)

[12] W.H. Fleming and W.M. McEneaney, Risksensitive control on an infinite horizon, SIAM J. Control and Optimization, Vol. 33, No. $6,1881 / 1915$ (1995)

[13] H. Ishii, H. Nagai and F. Teramoto, A singular limit on risk sensitive control and semi-classical analysis : Proceeding of the rth Japan-Russia Symp. on Prob. and Math. Stat., World Scientific, $164 / 173(1996)$.

[14] D.H. Jacobson, "Optimal stochastic linear systems with exponential performance criteria and relation to deterministic differential games", IEEE Trans. Automatic Control, vol. AC-18, $124 / 131(1973)$
[15] M.R. James, "Asymptotic analysis of nonlinear stochastic risk-sensitive control and differential games", Mathematics of Control, Signals and Systems, vol. 5, 401/417 (1992)

[16] M.R. James, "A partial differential inequality for dissipative nonlinear systems", Systems and Control Letters, vol. 21,315-320 (1993)

[17] M.R. James, J.S. Baras and R.J. Elliot, " Output Feedback Risk-snesitive Control and Differential Games for Continuous-Time Nonlinear Systems" 32nd IEEE CDC, San Antonio, (1993)

[18] H. Kaise and H. Nagai, Bellman-Isaacs equations of ergodic type related to risk-sensitive control and their singular limits, Asymptotic Analysis $16,347 / 362$ (1998)

[19] H. Kaise and H. Nagai, Ergodic type Bellman equations of risk-sensitive control with large parameters and their singular limits, Asymptotic Analysis 20,279/299 (1999)

[20] O.A. Ladyzhenskaya and N.N. Ural'tseva, Linear and Quasilinear Elliptic Equations, Academic Press, New York, (1968).

[21] W.M. McEneaney, "Connections between risksensitive stochastic control, differential games and $H_{\infty}$ control:the nonlinear case", Brown University $\mathrm{PhD}$ Thesis, (1993)

[22] H. Nagai, Bellman equations of risk-sensitive control, SIAM J. Control and Optimization, Vol. 34, No. $1,74-101$ (1996) .

[23] M. Reed and B. Simon, Methods of Modern Mathematical Physics, Vol. IV, Analysis of Operators, Academic Press, New York, (1978)

[24] T. Runolfsson, "Stationary risk-sensitive LQG control and its relation to LQG and $\mathrm{H}$ infinity control", Proc. 29-th CDC conference, 1018/1023 (1990)

[25] P. Whittle "Risk-sensitive linear quardratic Gaussian control" Adv. Appl. Prob., vol. 13, $764 / 777$ (1982)

[26] P. Whittle, "A risk sensitive maximum principle" Syst. Control Lett., vol. 16, 183/192 (1990)

[27] P. Whittle, "A risk snesitive maximum principle; The case of imperfect state information", IEEE Trans. Auto. Control, vol. 36, 793/801 (1991) 